\title{
Ultrafast ring-closing reaction dynamics of a photochromic furan-based difurylethene
}

\author{
A. Khodko ${ }^{\text {a }}$, V. Khomenko ${ }^{\text {a }}$, Y. Shynkarenko ${ }^{a}$, O. Mamuta $^{\text {a }}$, O. Kapitanchuk ${ }^{b}$, D. Sysoiev ${ }^{\text {c }}$, N. Kachalova ${ }^{\text {a }}$, \\ T. Huhn ${ }^{c}$, S. Snegir ${ }^{\mathrm{d}, *}$ \\ a Institute of Physics of NAS of Ukraine, 46 Nauky pros., Kyiv 03028, Ukraine \\ ${ }^{\mathrm{b}}$ Bogolyubov Institute for Theoretical Physics of NAS of Ukraine, 14-b, Metrolohichna str., Kyiv 03680, Ukraine \\ ${ }^{\mathrm{c}}$ University of Konstanz, Universitätsstr. 10, 78457 Konstanz, Germany \\ ${ }^{\mathrm{d}}$ Chuiko Institute of Surface Chemistry of NAS of Ukraine, 17 General Naumov str., Kyiv 03164, Ukraine
}

Keywords:

Diarylethenes

Photochromism

Photoswitching

Pump-probe

Femtosecond laser

TD-DFT

Absorption spectra

\begin{abstract}
A B S T R A C T
The ultrafast photoinduced ring-closing dynamics of a furan-based difurylethene (YnPhT) has been investigated by femtosecond transient absorption spectroscopy. We performed time-dependent density functional theory (TD-DFT) calculations to explain the experimental results in detail. The sub-picosecond time scale of the ring-closing reaction is comparable with thiophene-based analogues, but oxygen atoms at the photochromic core can avoid adverse interaction between switches and metal contacts in further applications. This observation proves that furan-based diarylethenes are potential optoelectronic elements with an ultrafast optical response.
\end{abstract}

\section{Introduction}

Photochromic molecules $[1,2]$, which can be reversibly triggered between a closed-ring (CF) and an open-ring (OF) form by alternating UV/VIS light illumination, are most promising elements for optoelectronic functional materials [3-6]. For this purpose, the reversible switching properties of the molecules must be preserved in various condensed phases $[7,8]$ as well as in connection to metal electrodes as well [9-11]. Ideally, both OF and CF of photochromic diarylethene derivatives (DAEs) should possess the following key properties [12]: thermal stability, high fatigue resistance, rapid optical response, and minor structural change while switching. DAEs completely meet these requirements, when their switching unit is functionalized with appropriate $\pi$-conjugated side-chain groups [13-15]. However, simultaneously these side-chain groups have an influence on optical properties [16-18], resistance to photodegradation [19] and on the dynamics of the ring closing/opening reaction [20-23]. Therefore, it is crucial to understand the relation between the structure of side-chain groups and ring closing/opening dynamics. Ultrafast pump-probe spectroscopy is a powerful tool to study the switching dynamics of DAEs at the

\footnotetext{
* Corresponding author.

E-mail address: ssnegir@gmail.com (S. Snegir).
}

picosecond time scale [24,25]. This method allows the timeresolved monitoring of the ultrafast processes that occur during the ring-closing reaction: redistribution of electronic density, molecular geometry changes, and $\pi-\pi^{*}$ conjugation at photochromic core [22]. Consequently, the combination of femtosecond time-resolved spectroscopy with TD-DFT calculations was found highly efficient for investigating of the ultrafast photoinduced ring-closing reaction $[21,26,27]$. Therefore, DAEs with various functional side chain groups were synthesized and are discussed in the literature recently [28-32], aiming at finding suitable side-chain groups to link DAEs with metallic electrodes. We recently developed furan-based diarylethenes with sulfur-free photochromic core and differently substituted $\pi$-conjugated side chains for application in single molecule-metal junction experiments [14,33]. Break-junction experiments [13] proved that $(1,2-$ bis(2-methyl-5-((4-(acetylthio)phenyl)ethynyl)-furan-3-yl) perfluorocyclopentene - YnPhT (Fig. 1) is suitable for implementation into organo-metallic hybrid-devices demonstrating strong fatigue resistance [15]. However, the influence of the $\pi$-conjugated 4(acetylthio) phenylethynyl side-chains of YnPhT and substitution of sulfur by oxygen (furan vs. thiophene) on the switching dynamics in contrast to a dithienyl DAE were not studied yet. In this work, we present investigation of the ring-closing reaction by femtosecond transient absorption spectroscopy and TD-DFT calculations. 


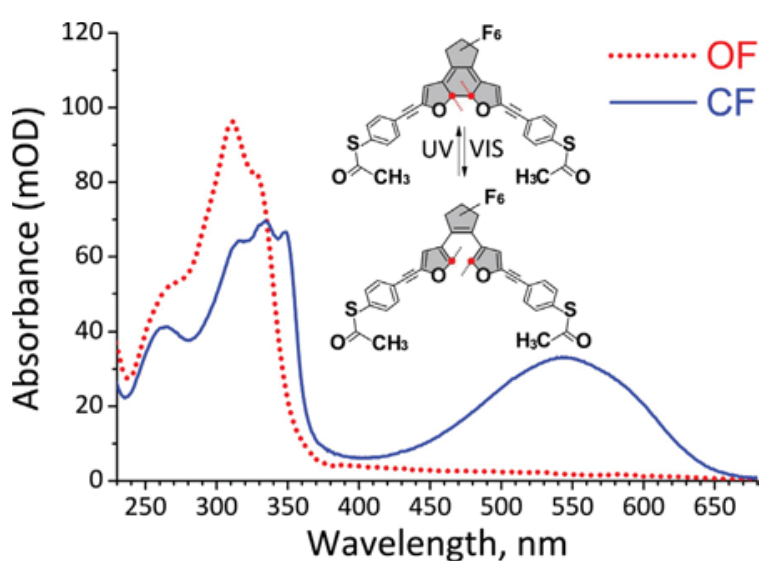

Fig. 1. Absorption spectra of $\mathrm{CF}$ (solid blue) and $\mathrm{OF}$ (dotted red) of YnPhT molecules in ethanol solution at $\left(C=5 \cdot 10^{-5} \mathrm{M}\right)$. Irradiation with ultraviolet light $(\lambda=250$ $350 \mathrm{~nm}$ ) leads to ring-closing reaction, while the reverse reaction of ring-opening occurs under visible light irradiation $(\lambda=450-650 \mathrm{~nm})$. The inset shows reversible structural changes of the bis-furylhexafluorocyclopentene photochromic core (gray) during UV/VIS illumination. (For interpretation of the references to colour in this figure legend, the reader is referred to the web version of this article.)

\section{Experiment}

The photoinduced dynamics were investigated by femtosecond transient absorption spectroscopy. The amplified femtosecond pulses $(E=0.5 \mathrm{~mJ}, \tau=150 \mathrm{fs}, \lambda=800 \mathrm{~nm}, v=1 \mathrm{kHz})$ from Ti:Sapphire laser (Coherent, USA) were split into two beams: pump initiated the ring-closing reaction, while probe tracked ultrafast changes in optical density. Since the ring-closing reaction occurs under UV illumination, we used the third harmonic generation ( $E=0.4 \mu \mathrm{J}, \tau=150 \mathrm{fs}, \lambda=266 \mathrm{~nm}, v=250 \mathrm{~Hz}$ ), obtained via the femtokit for third harmonic generation (Eksma Optics). As a probe radiation, the supercontinuum (SC, $\lambda=540-600 \mathrm{~nm}$ ), generated in an $\mathrm{Al}_{2} \mathrm{O}_{3}$ crystal by femtosecond pulses at $\lambda=800 \mathrm{~nm}$, was used. We probed the photoinduced optical density changes near the maximum of $\mathrm{CF}$ absorption band. The SC beam was split into the probe and the reference beams before the sample, then both beams were recorded by multichannel spectrograph (Imaging Spectrograph SP-2500i, Acton, USA). In order to determine the time delay range, the approximate zero position was obtained from mixing fundamental $\lambda=800 \mathrm{~nm}$ and SC in the nonlinear BBO crystal. In our data and figures the zero point position was chosen as the starting point of absorption changes. To correct SC fluctuations, the transient absorbance spectra were obtained by comparing the probe and the reference spectra for different time delays, and then, the group-velocity dispersion of SC pulse was corrected numerically at all obtained transient traces. YnPhT with concentration $\left(C=5 \cdot 10^{-5} \mathrm{M}\right)$ in ethanol was used. To avoid accumulation of CF molecules, the solution was pumped through a quartz flow-cell with $2 \mathrm{~mm}$ optical path and a buffer volume by a peristaltic pump with $2 \mathrm{ml} / \mathrm{s}$ flow rate. We irradiated the solution in the buffer volume by broad visible radiation (Schott KL $1500 \mathrm{LCD}, P=150 \mathrm{~W}$ ), which caused the reverse ring-opening reaction. This approach allowed refreshing of the solution in the cell with OF molecules by inducing ring-opening reaction of $\mathrm{CF}$ molecules. All studies were performed at room temperature.

\section{Results and discussion}

The absorption spectra of both, OF and CF of YnPhT (Fig. 1) show that $\mathrm{OF}$ undergoes the ring-closing reaction upon UV irradiation with $\lambda=250-350 \mathrm{~nm}$. As a result, the typical new absorption band of the CF grows in the range of $\lambda=450-650 \mathrm{~nm}$ wavelengths. This process can be easily followed by a drastic change in color from colorless to dark purple.

During the transient absorption measurements, the growth of optical density was observed at $\lambda=540-610 \mathrm{~nm}$ with a stepwise increase of delay between pump and probe pulses up to $0.8 \mathrm{ps}$ (Fig. 2a). After 0.8 ps, we detected only slight optical density fluctuation. No progressive spectral shifts during the first hundreds of femtoseconds after pump pulse are detected compared to other studies [22].

To characterize the gradual growth of the optical density, we determine the rise time as the time were absorbance changes from $10 \%$ to $90 \%$ of the final value of optical density. For initial data we obtained the rise time close to $420 \mathrm{fs}$. This time represents the upper boundary, because it reflects both, the transition time of the sample and the instruments response function. After reconvolution of sigmoid step function [37] and instrument response function, we found the upper boundary of the rise time is close to $350 \mathrm{fs}$ (cf. Supp. Inf. S1). Another approach [21,36] to characterize this process is fitting of the experimental data with convolution of exponential function and instrument response function that is shown at (Fig. 2b) (cf. Supp. Inf. S2). For the minimal expected instrument response function (FWHM $=212 \mathrm{fs}$ ), we obtained an upper boundary of the time constant of $200 \mathrm{fs}$. Further measurements with shorter laser pulses are required for a more precise determination of the ring-closing time constant.
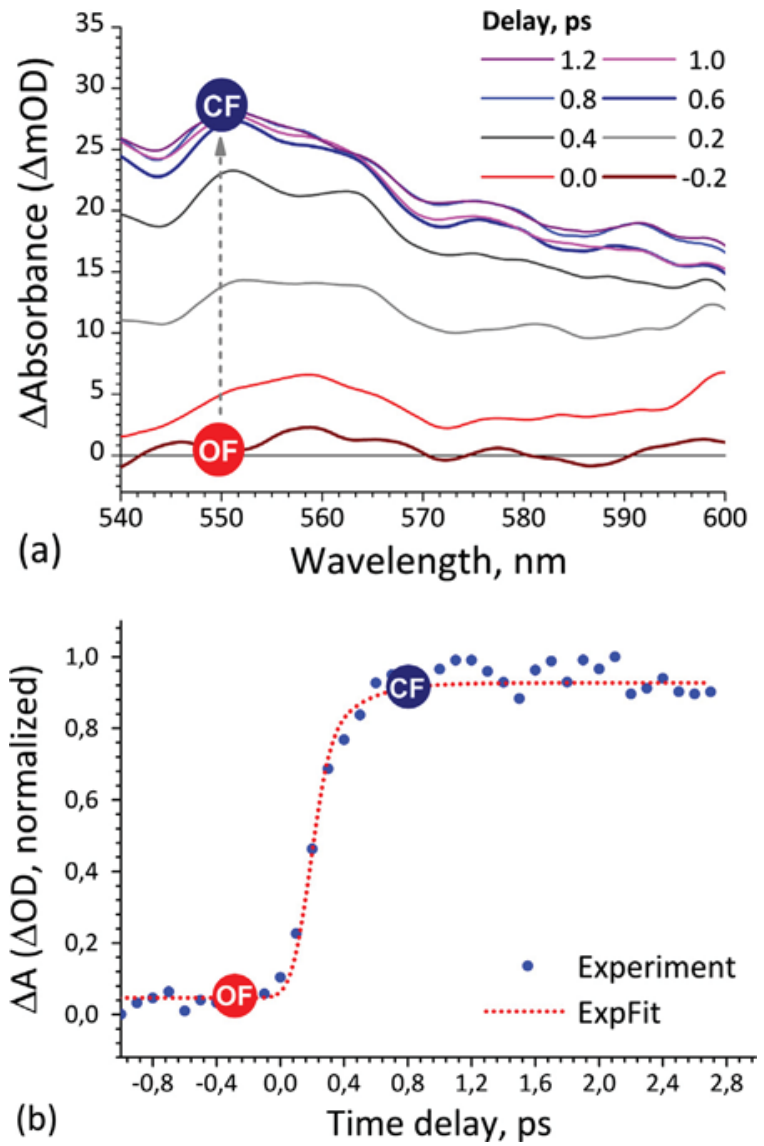

Fig. 2. Time-resolved spectra (a) displaying optical density changes during irradiation with $\lambda=266 \mathrm{~nm}$ to stimulate photoinduced ring-closing reaction. Points OF (red area) and CF (blue area) are highlighting the start and end-point of increased light absorbance; (b) - normalized time profile of ring-closing reaction extracted from time-resolved spectra at $\lambda=550 \mathrm{~nm}$ and fitted by reconvolution of exponential function and instrument response function (dotted line) (For details cf. Supp. Inf., Fig. S2). (For interpretation of the references to colour in this figure legend, the reader is referred to the web version of this article.) 
We assume that these rapid changes in optical density result from photoinduced electronic-density redistribution during the process of $\pi-\pi^{*}$ conjugation between reactive C-C atoms located at the photochromic core. Since, the form of optical density changes in subpicosecond timescale retrieves the characteristic absorption band of CF molecules (cf. Supp.Inf. S3). From timeresolved spectra, we obtained the time profile of the ring-closing reaction at the characteristic maximum absorption band of the $\mathrm{CF}$ at $\lambda=550 \mathrm{~nm}$ (Fig. 2b). It correlates well with general trends of ring-closing dynamics of diarylethene derivatives [21,36]. Reported ring-closing processes span times-ranges from hundreds of femtoseconds to hundreds of picoseconds. Different processes $[26,28]$ are involved that start from excitation of OF molecules, formation of $\pi-\pi^{*}$ conjugation at photochromic core, and finally thermal cooling of $\mathrm{CF}$ molecules.

To gain more detailed insight into the ring-closing reaction dynamics, we performed TD-DFT/B3LYP/6-31+G(d) calculations of the electronic structure evolution from the ground state to the correspondent excited states (Fig. 3) on the basis of the total energy changes of the isolated molecule. To verify the suitability of TDDFT for the analysis of switching dynamic, at first, we performed the calculation of main energetic states of the molecules. These states are responsible for the formation of the corresponding absorption band of CF and OF molecules (Fig. 1). The obtained data revealed good reproducibility of the shape of calculated spectra and experimental data (Fig. 3).

The $C F$ is characterized by a single band $(A)$ spanning the wavelengths $\lambda=450-650 \mathrm{~nm}$ and two intrinsic bands $(B, C)$ at $\lambda=240-$ $300 \mathrm{~nm}$ while the OF molecule has two bands $\left(B_{1}, C_{1}\right)$ in the UV region. The slight red-shift of the calculated $\mathrm{CF}$ absorption bands can be explained by solvent interaction effects and intermolecular interactions, which are not considered in TD-DFT calculations. Therefore, the relative values of the energy difference between ground state $S_{0}$ and corresponding excited state energy Sn calculated as the function of the distance of reactive $\mathrm{C}-\mathrm{C}$ carbon atoms will be used during the discussion. The calculated total energy profiles for ground $S_{0}$ and the first excited $S_{1}$ states as a function of the reaction coordinate are presented in Fig. 4.

The lowest values of relative energies of the ground state correspond to the molecules with $\mathrm{C}-\mathrm{C}$ distances between the reactive carbon atoms which are equivalent to $3.58 \AA$ in the $\mathrm{OF}$ and $1.53 \AA$ in the CF molecules. They are characterized by HOMOLUMO energy gap of $\Delta E=3.91 \mathrm{eV}$ and $\Delta E=2.23 \mathrm{eV}$ (Fig. 5), respectively. These values explain the nature of the correspondent absorption bands determined by UV absorption spectroscopy (Fig. 1). (OF: $\lambda=311 \mathrm{~nm}$, or $\Delta E=3.98 \mathrm{eV} ; \mathrm{CF}: \lambda=550 \mathrm{~nm}$, or $\Delta E=2.25 \mathrm{eV}$ ). The calculations demonstrate a robust potential

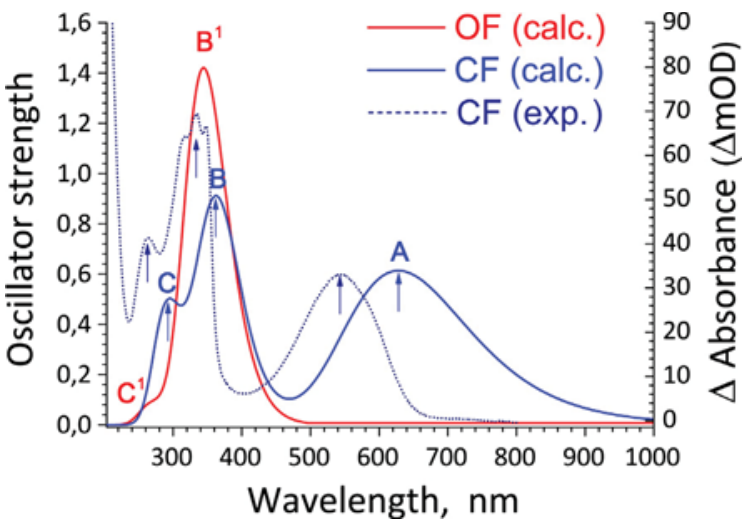

Fig. 3. Calculated (TD-DFT) spectra of isolated YnPhT molecules (solid curves) and measured absorbance spectra of YnPhT in ethanol (dotted curve).

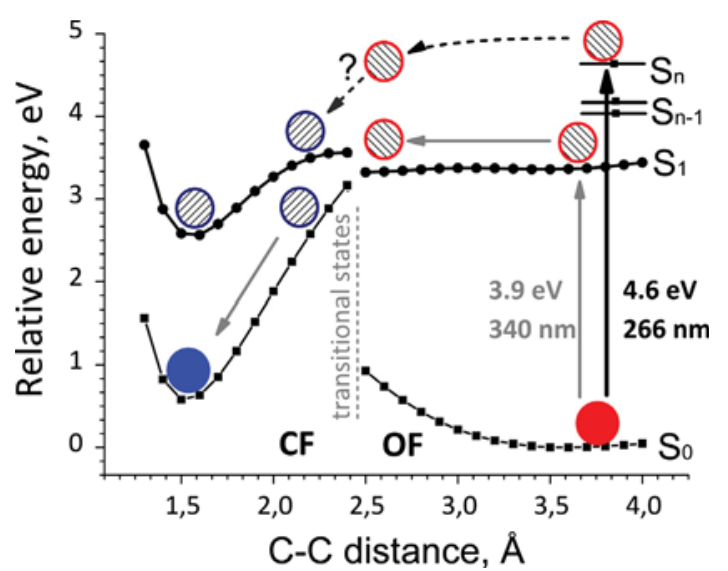

Fig. 4. Potential energy surfaces of ground state $S_{0}$ and first excited state $S_{1}$ for YnPhT in CF and OF calculated at TD-DFT/B3LYP/6-31+G(d) level. The geometry of single molecule was relaxed to the lowest energy configuration at each position (in the range of 1.3-4.0 $\AA$ with a step-width of $0.1 \AA$ ) along the C-C distance between the two carbon atoms of the photochromic core forming the central bond in the $\mathrm{CF}$. The positions of $S_{n}$ and $S_{n-1}$ energy levels are calculated for one C-C distance equivalent to $3.58 \AA$ for OF. The black arrows schematically represent the pathway of $\mathrm{YnPhT}$ after excitation $(\lambda=266 \mathrm{~nm})$ with following relaxation. The gray arrows represent conventional pathway of DAE CF formation when OF is excited by $\lambda=340 \mathrm{~nm}(3.9 \mathrm{eV})$. The energy barrier between the $\mathrm{S}_{1}$ excited states of OF and CF of DAEs was calculated to $0.77 \mathrm{eV}(17.8 \mathrm{kcal} / \mathrm{mol})$.

energy barrier of about $3 \mathrm{eV}$ separating $\mathrm{OF}$ and $\mathrm{CF}$ in the ground state $S_{0}$, which explains the thermal stability of YnPhT in solution and in thin films [15]. Switching barriers with a similar shape for difurylethene-based DAEs with different functional groups were obtained previously [33]. Thus, we conclude that replacing sulfur by oxygen (thiophene $\rightarrow$ furan) has no explicit influence on the height and shape of the switching barrier. Therefore, further explanation of obtained experimental results of (Fig. 2) can be done in the frame of existed theory of ring-closing reaction which includes several stages.

The preswitching stage is associated with the transition from the ground $\left(\mathrm{S}_{0}\right)$ to the corresponding excited electronic state, depending on the pump wavelength. In the current experiment YnPhT molecules are excited to Sn potential energy surface (Fig. 4). During this process, the redistribution of electronic orbitals from the side-chain groups to the photochromic core occurs, which is shown as the HOMO $\rightarrow \mathrm{LUMO}+n$ transition of OF molecules (Fig. 5). When an excited electronic state is reached (second stage), the ultrafast internal conversion starts within hundreds of femtoseconds and the $\mathrm{C}-\mathrm{C}$ distance between the reactive carbonatoms is becoming smaller. During this stage an excited molecule is relaxing along correspondent potential energy surface to the

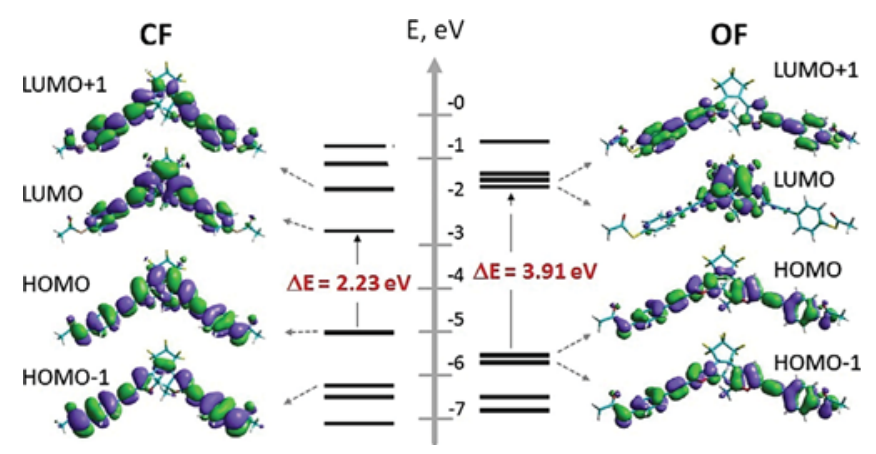

Fig. 5. Energy diagram of frontier HOMOs and LUMOs of the OF and CF of YnPhT molecules. 
transitional state (Fig. 4). As it was shown by Sendler et al. [34], a steeper energy curve towards the transitional state results in faster switching to the CF. This relaxation process becomes experimentally evident for certain types of molecules as an absorption band evolution in the visible wavelength regime, particularly as a progressive spectral shift during the first hundreds of femtoseconds after the pump pulse [22]. However, in the case of YnPht, no such spectral shift was detected in the monitored wavelength range, possibly since this electronic relaxation can occur even faster for furan-based diarylethenes during the first two hundreds of femtoseconds, which is close to resolution limit of our experimental setup. Instead, we assume that system is reducing the $\mathrm{C}-\mathrm{C}$ distance to around $2.5 \AA$ which is close to the transitional barrier. After that the progressive photoinduced ring-closing reaction occurs, leading to a strong increase of visible light absorption within $0.2-0.8 \mathrm{ps}$ after the initial UV irradiation pulse (Fig. 2). The further equilibrium ground state $S_{0}$ can be reached by internal conversion, which involves the redistribution of electronic orbitals between the photochromic core and the side-chain groups as a LUMO $\rightarrow$ HOMO transition of $\mathrm{CF}$ molecule.

Different reaction pathways (Fig. 4) in dependence of wavelength of the pump pulse were discussed in literature previously $[21,26]$. When the pump wavelength is close to the energy difference between the ground state $S_{0}$ and first excited $S_{1}$ state of OF (e.g., for YnPhT molecules a pump wavelength of $\lambda=340 \mathrm{~nm}$ is close to the energy gap near $\Delta E=3.9 \mathrm{eV}$ ), most authors $[7,20,22]$ conclude that the ring-closing reaction occurs in the subpicosecond time-regime through the conical intersection after fast electronic relaxation from the Franck-Condon states along the $S_{1}$ potential surface. The whole ring-closing process is then finalized by the vibrational relaxation of hot $\mathrm{CF}$ molecules during the next hundreds of picoseconds. However, when OF is pumped to higher excited states, in case of YnPhT by $\lambda=266 \mathrm{~nm}(\Delta E=4.6 \mathrm{eV})$, the relaxation pathway is not becoming obvious. Aloise et al. [26,27] proposed two pathways of ring-closing reaction occurring from higher excited states. The first one predicts the existence of a second conical intersection for the direct photoinduced ring-closing reaction without involving any already excited $\mathrm{CF}$ species. The second hypothesis assumes that when the ring-closing reaction has occurred the molecule is relaxing through the corresponding $S_{n}$ excited state of $C F$ with further ultrafast internal conversion to $S_{0}$ ground state $(\mathrm{CF})$. Due to limited time resolution of our instruments, it is difficult to distinguish between these two hypotheses so far. However, various advanced pump-probe techniques such as resonance enhanced femtosecond stimulated Raman scattering and femtosecond time-resolved fluorescence spectroscopy $[28,35]$ can provide additional information about ring-closing process in vicinity to transition state. The use of TD-DFT calculations is also limited and does not give any suitable information about what is happening when transitional state is reached. TD-DFT which being widely used for calculations of potential energy surfaces of the first excited states [7] can give only preliminary positions of higher potential energy surfaces when molecules are excited by $266 \mathrm{~nm}$. This uncertainty appears since the calculated density of states with energy gap close to $\Delta E=4.66 \mathrm{eV}(\lambda=266 \mathrm{~nm})$ is very high. (cf. Supp.Inf. Fig. S4). Therefore, such calculation do only provide qualitative information about transitions between possible molecular orbitals while quantitative estimations are still remaining inaccessible

\section{Conclusions and outlook}

Ultrafast photoinduced ring-closing reaction dynamics of a furan-based diarylethene with phenylethynyl side-chain groups equipped with protected thiol end-groups was studied by fem- tosecond transient absorption spectroscopy. The potential energy surfaces were calculated using TD-DFT approach to predict possible relaxation paths of YnPhT molecules when OF is excited by different wavelengths. Ultrafast spectroscopy revealed that the upper value of the rise time as the characteristic of the gradual growth of optical density during the ring-closing reaction of YnPhT molecules is close to $420 \mathrm{fs}$. By the use of an exponential function fit this time constant is determined to $200 \mathrm{fs}$. Independently from the used fitting functions, both of these values are smaller compared to thiophene based DAEs [20,22], revealing a faster ring-closing reaction of furan-based DAEs. We thus expect that replacing the photochromic cores thiophene with furan moieties might improve the optical properties of the molecules with regard to the cyclization dynamics. Therefore, on the basis of current observations and previous break-junction experiments we may assumed that the combination of phenylethynyl side-groups (acting as $\pi$ conjugated linkers) with furan-based photochromic core, might lead to excellent results when implemented in nanoresistor with optically controlled conductance operating on the sub- or picosecond timescale. However, the exact reason for this improvement of ring-closing dynamics of furan over thiophene-based DAE is presently unclear and requires additional experimental measurements to clarify relaxation pathway when molecules excited to $\mathrm{S}_{n}(\mathrm{OF})$ and more detailed theoretical studies with an emphasis on different excited configurations and nature of employed solvent [36]. Both of them may affect strongly the calculated switching barrier of DAEs.

\section{Acknowledgments}

The work was supported by the Grant of NAS of Ukraine for young scientists for realization of the research project, 20152016, and Deutsche Forschungsgemeinschaft through SFB767. Authors are grateful to the staff of the Center for collective use Femtosecond Laser Complex for useful discussions and measurements, and to Dr. D. Brida from the University of Konstanz for precise consultations on further experiments. Syntheses were performed by D.S. at University of Konstanz, D.S. was supported by Deutsche Forschungsgemeinschaft (DFG) project Hu 1682/3-1. The DFT calculations were performed by O.K. on the computer cluster at Bogolyubov Institute for Theoretical Physics of NAS of Ukraine, the work of O.K. was partially supported by the project No. 0116 U002067 of NAS of Ukraine.

\section{Appendix A. Supplementary material}

Supplementary data associated with this article can be found, in the online version, at http://dx.doi.org/10.1016/j.cplett.2016.12. 034.

\section{References}

[1] M. Irie, Photochem. Photobiol. Sci. 9 (2010) 1535-1542.

[2] M. Irie, Proc. Jpn. Acad. Ser. B. Phys. Biol. Sci. 86 (2010) 472-483.

[3] M.M. Russew, S. Hecht, Adv. Mater. 22 (2010) 3348-3360.

[4] J.M. Abendroth, O.S. Bushuyev, P.S. Weiss, C.J. Barrett, ACS Nano 9 (2015) 7746-7768.

[5] M.E. Gemayel et al., Nat. Commun. 6 (2015) 1-8.

[6] M. Irie, Chem. Rev. 100 (2000) 1685-1716.

[7] M. Irie, T. Fukaminato, K. Matsuda, S. Kobatake, Chem. Rev. 114 (2014) $12174-$ 12277.

[8] H. Jean-Ruel et al., J. Phys. Chem. B 117 (2013) 15894-15902.

[9] S. Snegir, I. Mukha, D. Sysoiev, E. Lacaze, T. Huhn, O. Pluchery, Materwiss. Werksttech. 47 (2016) 229-236.

[10] S.V. Snegir, P. Yu, F. Maurel, O.L. Kapitanchuk, A.A. Marchenko, Langmuir 30 (2014) 13556-13563.

[11] J. Wirth et al., J. Phys. Chem. C 119 (2015) 9.

[12] M. Irie, Springer, Japan, 2013, 298.

[13] Y. Kim et al., Nano Lett. 12 (2012) 3736-3742.

[14] B.M. Briechle et al., J. Nanotechnol. 3 (2012) 798-808. 
[15] D. Kim et al., Adv. Mater. 26 (2014) 3968-3973.

[16] S. Pu, C. Zheng, Z. Le, G. Liu, C. Fan, Tetrahedron 64 (2008) 2576-2585.

[17] R. Wang, X. Dong, S. Pu, G. Liu, Spectrochim. Acta A Mol. Biomol. Spectrosc. 137 (2015) 1222-1230.

[18] Q. Ai, S. Pang, K.H. Ahn, Chemistry 22 (2016) 656-662.

[19] M. Herder, B.M. Schmidt, L. Grubert, M. Patzel, J. Schwarz, S. Hecht, J. Am. Chem. Soc. 137 (2015) 2738-2747.

[20] S. Shim, I. Eom, T. Joo, E. Kim, K.S. Kim, J. Phys. Chem. A 111 (37) (2007) 89108917.

[21] P.R. Hania et al., J. Phys. Chem. A 106 (2002) 8498-8507.

[22] P.R. Hania et al., J. Phys. Chem. A 109 (2005) 9437-9442.

[23] K. Mori et al., J. Am. Chem. Soc. 113 (2011) 2621-2625.

[24] A.H. Zewail, Angew. Chem. Int. Ed. Engl. 39 (2000) 2586-2631.

[25] U. Megerle, I. Pugliesi, C. Schriever, C.F. Sailer, E. Riedle, Appl. Phys. B 96 (2009) $215-231$.
[26] S. Aloise et al., J. Am. Chem. Soc. 132 (2010) 7379-7390.

[27] S. Aloise et al., Phys. Chem. Chem. Phys. 16 (2014) 26762-26768.

[28] E. Pontecorvo, C. Ferrante, C.G. Elles, T. Scopigno, J. Phys. Chem. B 118 (2014) 6915-6921.

[29] R. Murata, T. Yago, M. Wakasa, J. Phys. Chem. A 119 (45) (2015) 11138-11145.

[30] S. Fredrich, R. Gostl, M. Herder, L. Grubert, S. Hecht, Angew. Chem. Int. Ed. Engl. 55 (2016) 1208-1212.

[31] T. Buckup, C. Sarter, H.-R. Volpp, A. Jaschke, M. Motzkus, J. Phys. Chem. Lett. 6 (2015) 4717-4721

[32] A. Khodko et al., Mol. Cryst. Liquid Cryst. 639 (01) (2016) 64-70.

33] D. Sysoiev et al., Chem. Eur. J. 17 (2011) 6663-6673.

[34] T. Sendler et al., Adv. Sci. 2 (2015) 1500017.

[35] T. Fukaminato et al., Photochem. Photobiol. Sci. 9 (2) (2010) 181-187.

[36] Y. Ishibashi et al., J. Am. Chem. Soc. 120 (2016) 1170-1177.

[37] L. Sporkel, J. Jankowska, W. Thiel, J. Phys. Chem. B 119 (2015) 2702-2710. 